\title{
SHORT COMMUNICATION \\ MICROBIOME AND DIVERSITY INDICES DURING \\ BLOOD STEM CELLS TRANSPLANTATION - NEW PERSPECTIVES?
}

\author{
Sergio Parco, Giulia Benericetti, Fulvia Vascotto, Giuseppina Palmisciano \\ Department of Advanced Technology, Institute for Maternal and Child Health, Trieste, Italy
}

\section{SUMMARY}

Objective: The human body is colonized by bacteria, fungi and viruses. Resident commensal bacteria are a fundamental line of resistance to colonization by exogenous microbes. They actively regulate the production of nutrients by the host through a negative feedback mechanism, in order to prevent the availability of nutrients for potential pathogens. While only a small fraction of these microorganism may be pathogenic, the relationship between host and commensal microbiome is now studied as a whole, impacting several aspects of the host biology. Some studies have made clear the progresses in examining the role of microbiome on transplants and graft versus host disease (GVHD) severity and its pathogenesis: the risk of complications from allogenic hematopoietic stem cells transplantation (HSCT) is greater with the highest mortality if a patient has a lower bacterial diversity in the gut prior to the transplantation process beginning. Microbiota-associated molecular patterns are directly recognized by pathogen recognition receptors. The development of molecular methods has greatly expanded our knowledge of the composition and function of the microbiome in health and disease, shortening the response times vs. microbiological culture tests. The gut flora can make the difference when it comes to allo-HSCT. The aim of the study was to monitor microbiome of 10 children during allo-HSCT.

Methods: Oral specimens and gut faecal microbiome (100 grams) samples were collected at 2, 16, 24 days. The samples were analysed by polymerase chain reaction and primary sequencing was done. To calculate the biodiversity of microbiome the Shannon index and the Observed species index were chosen.

Results: Our study suggests some differences in the diversity indices (DIs) in 5 children affected by GVHD vs. not affected. The Dls in oral and faecal specimens show in all patients a diminution in the post-transplant phase with an improvement in species diversity after 16 days from the transplant. The Observed species index in faeces specimens after 16 days was higher in patients which had not GVHD; moreover, patients with GVHD showed a deterioration at 24 days. Oral specimens after 24 days showed a parallel trend in the two groups. The Shannon index shows a downward trend in faeces specimens of the children with GVHD at 24 days; the children without GVHD recover a good trend of entropy. Oral specimens at 24 days show low entropy in the two groups. Very aggressive bacterial species as Cronobacter and Routella in the faeces specimens of a child had not serious consequences for disease status: Cronobacter were not present 24 days after transplantation

Conclusions: The data show the microbial metabolome could have an impact on patients with GVHD vs. no GVHD. A better understanding of the role of the oral and gut microbiome in GVHD can give directions to move towards the development of innovative approaches for preventing GVHD following allo-HCT, reducing also antibiotic therapy.

Key words: oral and gut microbiome, blood stem cells transplantation, graft versus host disease

Address for correspondence: S. Parco, Department of Advanced Technology, Institute for Maternal and Child Health, Via dell'Istria 65/1, 34137, Trieste, Italy. E-mail: info@sergioparco.it

https://doi.org/10.21101/cejph.a5393

\section{INTRODUCTION}

Different bacterial populations colonize the gastrointestinal tract modulating host inflammation and immune tolerance (1, 2). Their concentration is very high in the oral cavity: it consists mainly of anaerobes such as Prevotella spp. and Peptostreptococcus. The gut flora instead, as Bacteroides, Fusobacterium and Eubacterium provides a multitude of bacterial genres: in particular, Gram-negative Bacteroides and Gram-positive Actinobacteria. They secrete a multitude of metabolites that affect host immunity and biology. The diversity indices (Dis) are methods to study the variability and composition of a habitat or community, describing the number of species and the degree of their distribution in the community (3).

Allogenic hematopoietic stem cells transplantation (alloHSCT) is a potentially curative treatment for many patients who would otherwise succumb to haematological malignant disease and immune disorders. It is associated with a variety of complications, including infections, graft versus host disease (GVHD) and inflammation, as commonly seen in the liver, lung, skin and intestine, increasing the mortality after allo-HSCT (4). During allo-HSCT, the gastrointestinal mucosa is damaged, and 
colonizing bacteria are impacted, leading to an impaired intestinal microbiota with reduced diversity. The DIs are particularly impacted by allo-HSCT, being both the origin and the target of many post-transplant complications, and they have a special role in the protection of the host from $\operatorname{GVHD}(5,6)$.

Many recent studies have made strides in examining the taxonomic composition and activity of the host and donor microbiome. New publications examine the role of microbial metabolites and the immunotherapy in the homeostasis of host physiology as cause of gut dysbiosis and to prevent the onset of disease. Nutritional protocols are continually established with the hope that new therapies may be developed soon to halt disease progression (7). The use of recently developed molecular methods has greatly expanded our knowledge of the composition and function of the human microbiome during health and disease, shortening the response times vs. microbiological culture tests. The PCR technologies employed for these studies are too expensive, compared to traditional diagnostic microbiological tests, which are reserved for routine checks; however, all new technologies are expensive in the beginning and this experience is a pilot study of our Institute to suggest new translated cares and therapies (2). The aim of this work was to study the oral and gut microbiome DIs, during alloHSCT stem cell transplantation, in 10 children affected by acute myeloid leukemia, of which 5 had developed GVHD.

\section{MATERIALS AND METHODS}

This experience was performed monitoring two DIs, during transplant period, at 2, 16, 24 days; 10 children between 3 and 10 years of age have been studied, of which 5 had developed GVHD. They were hospitalized at the Institute for Maternal and Child Health of Trieste (a town of Friuli Venezia Giulia, a region of Northeastern Italy). Two types of samples, oral and faecal specimens, were collected for each patient. To calculate the biodiversity of microbiome we have chosen two DIs: the Shannon index and the Observed species index.

The Shannon index is an information statistic index; it assumes that all species are represented in a sample and that they are randomly sampled. It is a measure of entropy in a community and integrates information on the richness and uniformity of an ecosystem. It can vary from 0 to infinity. The Observed species index describes instead the uniqueness of the Operational Taxonomic Units (OTUs) present in each sample. Specifically, OTUs are used to classify bacteria based on sequence similarity; in 16s metagenomic approaches, OTUs are clusters of similar sequence variants of the 16s rDNA marker gene sequence. Each of these clusters is intended to represent OTUs of a species or genus of bacteria based on the sequence similarity threshold.

The study had been approved by the Bioethics Committee of the Institute and the informed consent had been obtained from parents of the patients.

\section{DNA Extraction}

Gut faecal microbiome (100 grams) sample were subjected to pre-lysis, using lysis buffer easyMAG ${ }^{\circledR}$ (Biomerieux, North Carolina, USA) and proteinase K 20 micrograms/milliliter and centrifuged for 10 minutes at $13,000 \mathrm{rpm}$.
The DNA were extracted from the pre-lysed champion using the NucliSENS ${ }^{\circledR}$ easyMAG ${ }^{\circledR}$ semiautomatic system (Biomerieux, North Carolina, USA): the extraction system exploits the tendency of nucleic acids to adsorb to silica particles in the presence of a chaotropic salt. In order to evaluate the purity and quality of the extracted material, a reading was performed with the Nanodrop 100 (Thermo Scientific, Massachusetts, USA).

\section{Amplification}

The next step was the preparation of the library, which is the amplification of a genome target region.

This phase is divided into two steps consisting of the execution of two sequential Real Time PCRs.

Phase 1: first PCR I amplification.

A first quantitative PCR (CFX96 Deep Well Bio-Rad, California, USA) with EvaGreen probe (Biotium, California, USA) was performed to amplify the $400 \mathrm{pb}$ V1-V3 target gene of the rRNA 16S gene using 27FYM primers (5 '-AGRGTTYGATYMTGGCTCAG-3') and U534R (5'-ATTACCGCGGCTGCTGG-3'). The reaction mix was prepared in low retention tubes for a final volume of $10 \mu \mathrm{l}$, composed of $8 \mu \mathrm{l}$ of mix and $2 \mu \mathrm{l}$ of DNA, using the KAPA Library Amplification kit (Kapa Biosystems, Massachusetts, USA).

Phase 2: second PCR_II amplification.

Starting from the first ampere (PCR_I) a second Real Time PCR was performed, making a $1: 100$ dilution for a total volume of $10 \mu \mathrm{l}$. It is a nested PCR in which the primers amplify the V3 region of the 16S RNA 200 pb length. Primers used were B338F_trP1 (5'-ACTCCTACGGGAGGCAGC-3') and U534R_BC_A. Both contained a complementary adapter, used on the magnetic spheres for PCR in emulsion: the primer U534R_BC_A, contained a barcode corresponding to a short sequence of nucleotides that differs from sample to sample and allows their characterization. The thermal profile was shown on the graph.

\section{Electrophoresis on Agarose Gel}

The quality and length of the amplification products were verified by $2 \%$ agarose gel electrophoresis (p/v, 1989).

The gel was immersed in a TBE1X run buffer (TBE10X: $108 \mathrm{~g}$ Tris/Base, $55 \mathrm{~g}$ of boric acid, $40 \mathrm{ml}$ EDTA $0.5 \mathrm{M} \mathrm{pH} 8$ ) and it was subjected to an electric field of $100 \mathrm{~V}$ at room temperature for a variable time. At the end of the run the DNA has been visualized by colouring with Gel Red (Biotium, California, USA) diluted $1: 10000$. For this step another DNA intercalator was used, the Midori (Nippon Genetics Europe, Germany).

\section{Quantification and Pooling of the Library}

For the library quantification, the Qubit ${ }^{\circledR}$ 2.0 Fluorometerspectrophotometer was used, with the Qubit ${ }^{\circledR}$ dsDNA BR Assay Kit (Life Technologies, Massachusetts, USA); this assay is highly selective for the measurement of dsDNA. The quantification was performed because an equal quantity of each PCR_II must be used to obtain a total solution (pooled library), with a final concentration of $26 \mathrm{pM}$, as it is required by the sequencing protocol. 


\section{PCR in Emulsion}

The emulsion PCR was performed using the pooled library through the Ion OneTouch2 system with the Ion PGMTM Template OT2 200 Kit (Life Technologies, Massachusetts, USA). During this first pass the nucleic acid fragments of the library were bound to magnetic beads by the adapters (A and trP1). This system is based on the compartmentalization of DNA in a water/ oil emulsion. The fragments were segregated in the small drops of water of the emulsion and then were amplified.

For the second step we used the OneTouch Enrichment System for the marbles selection and the amplification following the provided protocol (Ion PGMTM Template OT2 200 Kit, Life Technologies, Massachusetts, USA).

\section{Chip Loading and Sequencing}

Sequencing was performed with the Ion PGM system using the Ion PGM TM Sequencing 200 Kit v2 kit (Life Technologies, Massachusetts, USA). The Ion 316v2 chips (Life Technologies, Massachusetts, USA), with a capacity of 200-250 Mb, was chosen for this step.

\section{Data Analysis}

The sequencing analysis of the PCR products obtained by amplification of $200 \mathrm{pb} \mathrm{V1-V3} \mathrm{target} \mathrm{gene} \mathrm{of} \mathrm{the} \mathrm{rRNA} \mathrm{16S} \mathrm{gene,}$ was performed by bioinformatics technicians using the Qiime 1.8 .0 software. For the alignment of the sequences, the reference databases were Greengenes (release 13.8) and M5RNA. The 16S rRNA of $200 \mathrm{pb}$ length was analyzed, lower length was discarded. The uncodified version used of the classic t-test, that provides a way to compare the two groups faeces and oral specimens, had no statistical significance.

\section{RESULTS}

Our study suggests some differences in the diversity indices (DIs) in 5 children affected by GVHD (Figures 1,2) vs. not affected (Figures 3, 4).

The DIs in oral and faecal specimens show in all patients a diminution in the post-transplant phase with an improvement in species diversity after 16 days from the transplant.

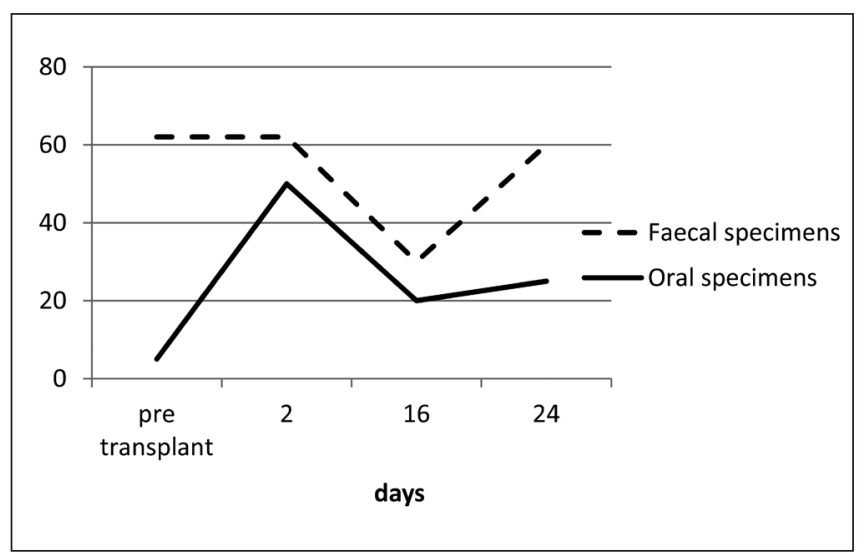

Fig. 1. Observed species index of 5 children during allo-HSCT.

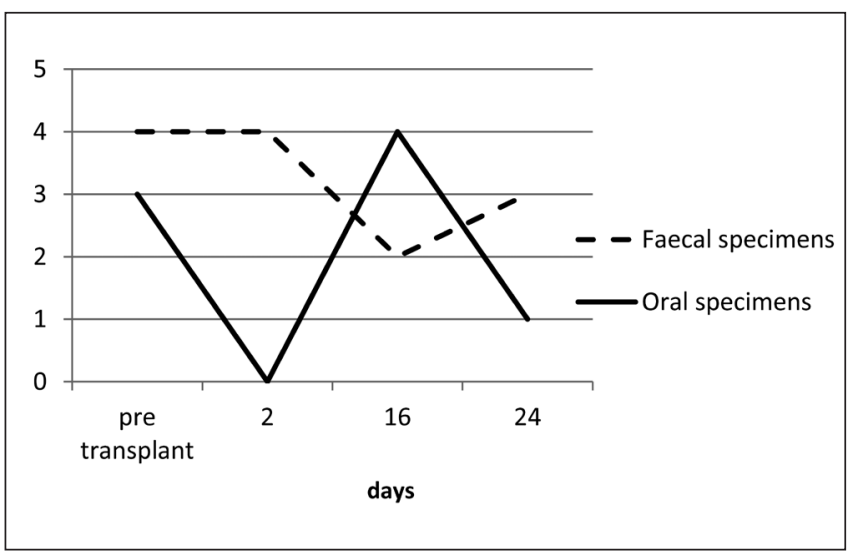

Fig. 2. Shannon index of 5 children during allo-HSCT.

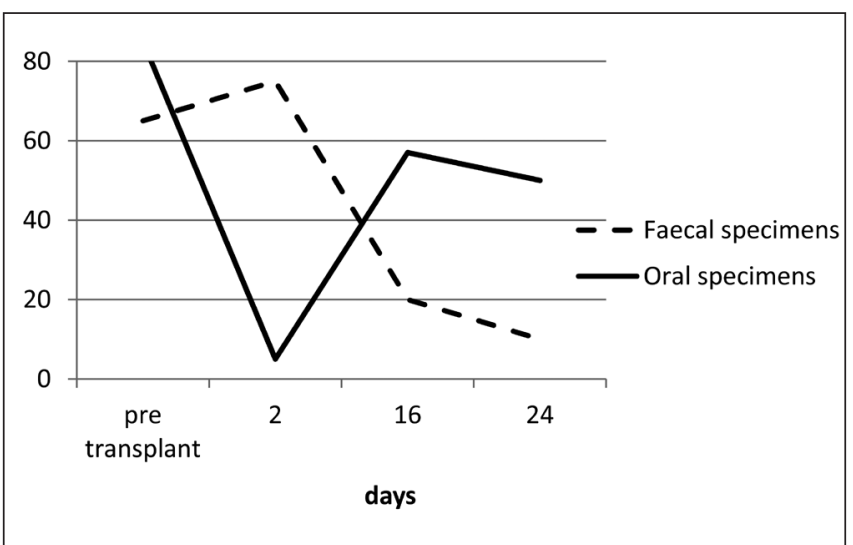

Fig. 3. Observed species index of 5 children with GVHD during allo-HSCT.

The Observed species index in faeces specimens after 16 days was higher in patients which had not GVHD; moreover, patients with GVHD show a deterioration at 24 days. Oral specimens after 24 days show a parallel trend in the two groups (Figures 1, 3).

Shannon index shows a downward trend in faeces specimens of the children with GVHD at 24 days; the children without GVHD recover a good trend of entropy. Oral specimens at 24 days show low entropy in the two groups (Figures 2, 4).

In a child who developed GVHD, the loss of this diversification and the appearance of very aggressive bacterial as Cronobacter and Routella in the faeces specimens, had not serious consequenc-

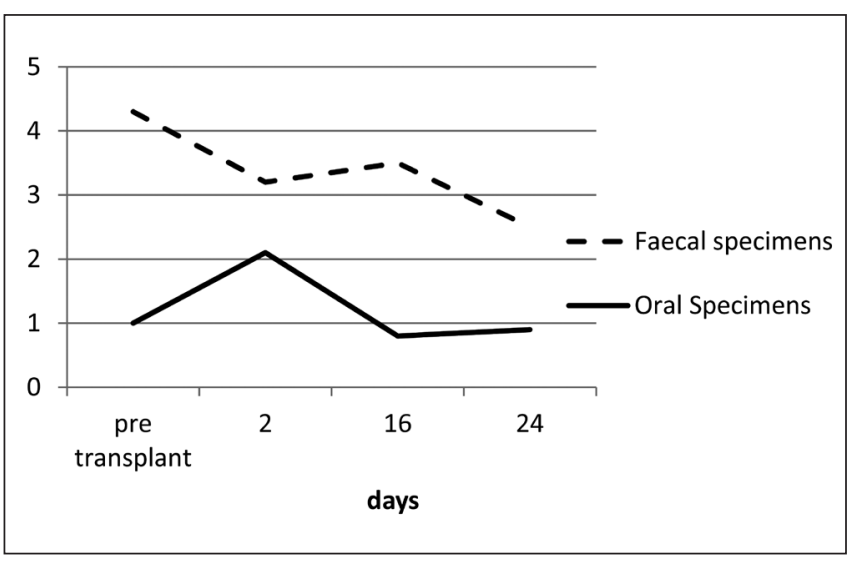

Fig. 4. Shannon index of 5 children with GVHD during allo-HSCT. 


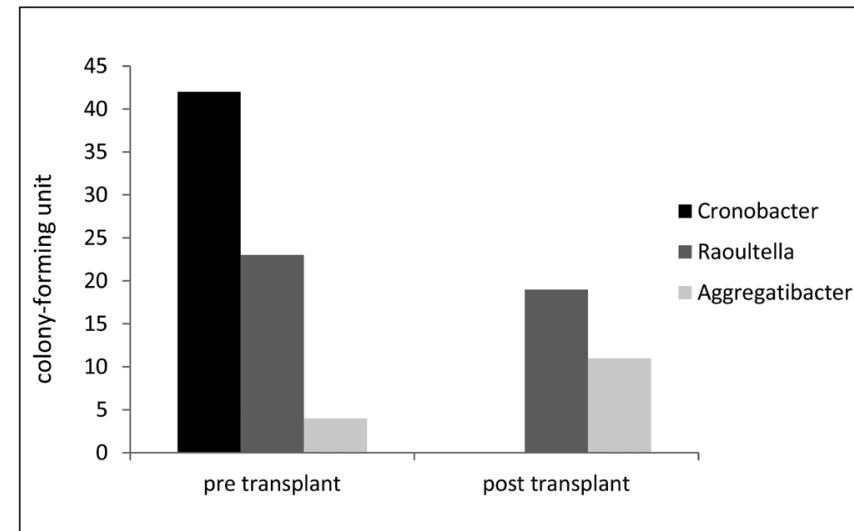

Fig. 5. Cronobacter and other Bacteroides levels during alloHSCT in a child with GVHD (faecal specimens).

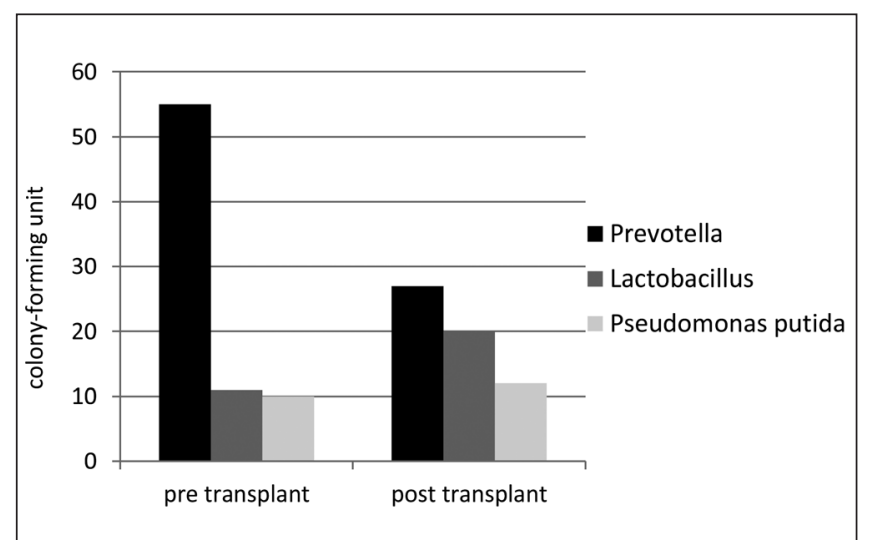

Fig. 6. Pseudomonas putida and other Bacteroides levels during allo-HSCT in a child with GVHD (faecal specimens).

es for disease status: Cronobacter were not present 24 days after transplantation (Fig. 5). In another child with GVHD, the presence of Pseudomonas putida was considered a low-virulence pathogen and it was recognized as no cause of bacteraemia (Fig. 6).

\section{CONCLUSIONS}

Many authors describe the different factors that influence the successful outcome of a transplant $(8,9)$. Among these it is necessary to consider the intestinal microbiota: the close relationship between the bacteria that colonize the body and the immune system seems to play an important role in reducing infections and rejection reactions of the new organ $(10,11)$.

Researches have tried to clarify the relationships between transplants, immune response and microbiome $(12,13)$. The main cause of post-transplant mortality and complications is the infection by pathogens, which finds a favourable environment in an immunosuppressed organism (14). The stem cell transplantations and their complications result from an interaction between the human host and the microbiome; variations in human responses to the microbiome are still little known and the influence of environmental factors introduces a further complexity layer (15).

The risk of infection and GVHD is associated also with various environmental and genetic factors, and more recently it is hypothesized that the composition of the microbiome may be linked to post-transplant complications $(16,17)$. The influence of the microbiome on the immune function demonstrates that the biodiversity influences not only the proliferation of opportunistic pathogenic bacteria, but also the effectiveness of anti-GVHD therapies: in the presence of some aggressive bacterial species it is necessary to modify the dosage of immunosuppressive drugs (18).

The gene amplification technologies that we used in this experience, and the outcomes of the patients, showed in real time the changes of microbiota during the post-transplant period, without waiting for the cultural responses (19-21). In patients who developed GVHD the loss of diversity and the appearance of very aggressive bacterial as Cronobacter can have serious consequences on a disease condition development (22).

Inflammatory pathologies can be caused by immunosuppressive drugs, and cause imbalances in the microbiome; the differences found before and after bone marrow and stem cell transplantation, in adult and paediatric patients, can be used as predictive tools to determine the possible long-term transplant outcome (23). Recently it has been shown that also the exposure to some antigens from the transplanted organ causes an immune response mediated by the microbiome (24). Although their mechanism of action has been poorly clarified, some bacterial strains appear to positively modify the composition of the microbiome, favouring a series of positive effects on the immune system; in addition, an increasing number of studies suggest the administration of probiotics as an alternative therapy (25).

We think a better understanding of the relationship among microbiome, immune system and transplantation complications will help to set up targeted strategies to increase graft tolerance and decrease the burden of immune reactions (26). Our experience on 10 paediatric cases confirms the speed and specificity of the tests used, which have contributed to an effective monitoring and predicting of transplant complications; microbiome has been studied shortening the response times of microbiological culture tests.

About the high cost-effective and high-throughput of PCR analysis vs. traditional microbiological tests (culture-based assays for confirmation), this is compensated by a more quickly care. The authors consider that all new technologies, at the beginnings, are expensive, then the use decreases their cost and that all methods have both advantages, drawbacks and limitations. In the near future the modern molecular methods will supersede conventional old techniques. Technical advances in DNA and RNA sequencing will provide vast amounts of new data. Increased understanding of the vital role of the gut microbiome in GVHD can give directions to move towards the development of improved innovative approaches for preventing or treating GVHD following allo-HCT, with research programmes and the promotion of multicentre surveillance studies.

\section{Conflict of Interests}

None declared

\section{REFERENCES}

1. Staffas A, Burgos da Silva M, van den Brink MR. The intestinal microbiota in allogeneic hematopoietic cell transplant and graft-versus-host disease. Blood. 2017;129(8):927-33.

2. van Belkum A, Durand G, Peyret M, Chatellier S, Zambardi G, Schrenzel $\mathrm{J}$, et al. Rapid clinical bacteriology and its future impact. Ann Lab Med. 2013;33(1):14-27. 
3. O'Hara AM, Shanahan F. The gut flora as a forgotten organ. EMBO Rep. 2006;7(7):688-93.

4. Holler E, Butzhammer P, Schmid K, Hundsrucker C, Koestler J, Peter K, et al. Metagenomic analysis of the stool microbiome in patients receiving allogeneic stem cell transplantation: loss of diversity is associated with use of systemic antibiotics and more pronounced in gastrointestinal graftversus-host disease. Biol Blood Marrow Transplant. 2014;20(5):640-5.

5. Biagi E, Zama D, Nastasi C, Consolandi C, Fiori J, Rampelli S, et al Gut microbiota trajectory in pediatric patients undergoing hematopoietic SCT. Bone Marrow Transplant. 2015;50(7):992-8.

6. Penders J, Thijs C, Vink C, Stelma FF, Snijders B, Kummeling I, et al. Factors influencing the composition of the intestinal microbiota in early infancy. Pediatrics. 2006;118(2):511-21.

7. Mountzouris KC, McCartney AL, Gibson GR. Intestinal microflora of human infants and current trends for its nutritional modulation. Br J Nutr. 2002;87(5):405-20.

8. Mathewson N, Reddy P. The microbiome and graft versus host disease. Curr Stem Cell Rep. 2015;1(1):39-47.

9. Quigley EM. Microflora modulation of motility. J Neurogastroenterol Motil. 2011;17(2):140-7.

10. Barbara G, Stanghellini V, Brandi G, Cremon C, Di Nardo G, De Giorgio $\mathrm{R}$, et al. Interactions between commensal bacteria and gut sensorimotor function in health and disease. Am J Gastroenterol. 2005;100(11):2560-8.

11. Perry GH, Dominy NJ, Claw KG, Lee AS, Fiegler H, Redon R, et al Diet and the evolution of human amylase gene copy number variation. Nat Genet. 2007 Oct;39(10):1256-60.

12. Ohman L, Simren M. Pathogenesis of IBS: role of inflammation, immunity and neuroimmune interactions. Nat Rev Gastroenterol Hepatol. 2010;7(3):163-73.

13. Spiller RC. Infection, immune function, and functional gut disorders. Clin Gastroenterol Hepatol. 2004;2(6):445-55.

14. Mancini N, Greco R, Pasciuta R, Barbanti MC, Pini G, Morrow OB, et al. Enteric markers as early predictors of clinical outcome in allogenic hematopoietic stem cell transplant: results of a prospective study in adult patients. Open Forum Infect. Dis. 2017;4(4):215-17.

15. Zama D, Biagi E, Masetti R, Gasperini P, Prete A, Candela M, et al. Gut microbiota and hematopoietic stem cell transplantation: where do we stand? Bone Marrow Transplant. 2017 Jan;52(1):7-14.

16. Khosravi A, Yáñez A, Price JG, Chow A, Merad M, Goodridge HS, et al. Gut microbiota promote hematopoiesis to control bacterial infection. Cell Host Microbe. 2014;15(3):374-81.

17. Iida N, Dzutsev A, Stewart CA, Smith L, Bouladoux N, Weingarten RA, et al. Commensal bacteria control cancer response to therapy by modulating the tumor microenvironment. Science. 2013;342(6161):967-70.

18. Taur Y, Xavier JB, Lipuma L, Ubeda C, Goldberg J, Gobourne A, et al. Intestinal domination and the risk of bacteremia in patients undergoing allogeneic hematopoietic stem cell transplantation. Clin Infect Dis. 2012;55(17):905-14

19. Zwielehner J, Lassi C, Hippe B, Pointner A, Switzeny OJ, Kitzweger E, et al. Changes in human fecal microbiota due to chemiotherapy analyzed by TaqMan-PCR, 454 sequencing and PCR-DGGE fingerprinting. PloS One. 2012;6(12):e28654. doi: 10.1371/journal.pone.0028654.

20. Taur Y, Jenq RR, Perales M-A, Littmann ER, Morjaria S, Ling L, et al. The effects of intestinal tract bacterial diversity on mortality following allogeneic hematopoietic stem cell transplantation. Blood. 2014;124(7):117482.

21. Kalliomaki M, Collado MC, Salminen S, Isolauri E. Early differences in fecal microbiota composition in children may predict overweight. Am J Clin Nutr. 2008;87(3):534-8.

22. Chen Y, Zhao Y, Cheng Q, Wu D, Liu H. The role of intestinal microbiota in acute graft-versus-host disease. J Immunol Res. 2015;2015:145859. doi: $10.1155 / 2015 / 145859$

23. Peled JU, Devlin SM, Staffas A, Lumish M, Khanin R, Littmann ER, et al. Intestinal microbiota and relapse after hematopoietic-cell transplantation. J Clin Oncol. 2017;35(15):1650-9.

24. Andermann T, Peled J, Ho C, Reddy P, Riches M, Storb R, et al. Microbiome-host interactions in hematopoietic stem cells transplant recipients. Bio Blood Marrow Transplant. 2018;24(7):1322-40.

25. Andermann TM, Rezvani A, Bhatt AS. Microbiota manipulation with prebiotics and probiotics in patients undergoing stem cell transplantation. Curr Hematol Malig Rep. 2016;11(1):19-28.

26. Tawara I, Liu C, Tamaki H, Toubai T, Sun Y, Evers R, et al. Influence of donor microbiota on the severity of experimental graft-versus-hostdisease. Biol Blood Marrow Transplant. 2013 Jan;19(1):164-8.

27. Knight $\mathrm{P}$, Campbell BJ, Rhodes JM. Host-bacteria interaction in inflammatory bowel disease. Br Med Bull. 2008;88(1):95-113.

28. Jenq RR, Ubeda C, Taur Y, Menezes CC, Khanin R, Dudakov JA, et al Regulation of intestinal inflammation by microbiota following allogeneic bone marrow transplantation. J Exp Med. 2012;209(5):903-11.

29. Jenq RR, Taur Y, Devlin SM, Ponce DM, Goldberg JD, Ahr KF, et al. Intestinal blautia is associated with reduced death from graft-versus-host disease. Biol Blood Marrow Transplant. 2015 Aug;21(8):1373-83.

30. Golob JL, Pergam SA, Srinivasan S, Fiedler TL, Liu C, Garcia K, et al. Stool microbiota at neutrophil recovery is predictive for severe acute graft versus host disease after hematopoietic cell transplantation. Clin Infect Dis. 2017;65(12):1984-91.

Received April 18, 2018 Accepted in revised form June 24, 2019 